\title{
High-frequency Power Generation and Distribution in Multi-megawatt Power Systems
}

\author{
J.H. Beno, R.E. Hebner, and A. Ouroua \\ Center for Electromechanics \\ The University of Texas at Austin \\ Austin, USA
}

\begin{abstract}
A model of an autonomous power system that generates and distributes power at $240 \mathrm{~Hz}$ has been developed. This high-frequency ac power system (HFAC) includes several gas turbine-generator units, two propulsion power trains, several power distribution and conversion units, a supercapacitor energy storage module, a high-power pulse load, and multiple service loads. Four case studies are considered. These are: partial loss of generation, application of a large step load, power restoration to a vital load, and operation of energy storage. Preliminary analysis shows that the power system remains under control during the four events. Some benefits of the HFAC architecture are highlighted first and analysis results of the case studies follow.
\end{abstract}

\section{INTRODUCTION}

An effective power system has to be tailored to loads it supplies and the environment in which it operates. In applications where size, efficiency, stability, controllability, and survivability are the most important factors, autonomous power systems do not have to conform to conventional architectures where $60 \mathrm{~Hz}$ and multi-kilovolt power is generated and transmitted long distances to minimize losses. Rather, self-contained power systems where generation and loads are at proximity of each other, transmission cables are short and therefore the need for a low transmission frequency to keep line reactance low is no longer a factor, and frequencies higher than $60 \mathrm{~Hz}$ are possible. In addition, lower ohmic losses in the short cables allow operations at lower voltage levels which are now limited only by power conversion modules needed to supply diverse loads as well as personal safety considerations. Considerations like these have led to $400 \mathrm{~Hz}$ power systems in aircrafts and dc systems in automobiles.

The determination of the optimum generation frequency and the distribution voltage requires a detailed analysis of the load profiles and operational conditions since desirable properties such as small size, high efficiency, and low cost often compete with each other. A compromise that aims at balancing these requirements, along with environmental considerations, determines the appropriate power system architecture. This architecture may include high-frequency generation, medium voltage ac and dc distribution, compact high-frequency transformers, and various power conversion modules that are best suited to supply power to specific loads.

In this paper, we focus on one aspect of this architecture, mainly, high-frequency generation and distribution with application to an all-electric ship.

\section{SIZE-REDUCTION ADVANTAGES}

\section{A. High-speed and multi-pole generators}

The output power of synchronous generators is directly proportional to speed, the square of the air-gap diameter, and the active length among other factors such as the air-gap flux density and line current density. This power-speed-size dependence indicates that for a given power level, increasing the speed reduces the size of the machines. This size reduction is limited, however, by structural and rotor dynamics considerations. Nevertheless, significant reduction can be achieved with high speeds [1] which results in high-frequency power. In addition, for a given speed, increasing the number of poles will also increase the electrical frequency. This is relevant when several generating units running at different speeds need to be connected in parallel, requiring a common frequency. Increasing the number of poles from 2 to 4 for example reduces the back-iron needed in the machine magnetic circuit and therefore reduces the size, although to a lesser extent than a direct speed increase.

\section{B. High-speed, directly-coupled, turbo-generators}

Power systems that use gas turbines as prime movers can achieve significant size reduction and improve efficiency by directly coupling the gas turbines to the generators, i.e. without the use of gear boxes [2], [3]. This topology is more advantageous for low to medium power levels (1-15 MW) because gas turbine speed is higher at lower power, as illustrated in Fig. 1 [2]. At low to medium power a doubleadvantage can be achieved: removal of the gear boxes and size-reduction of the high-speed generators. 


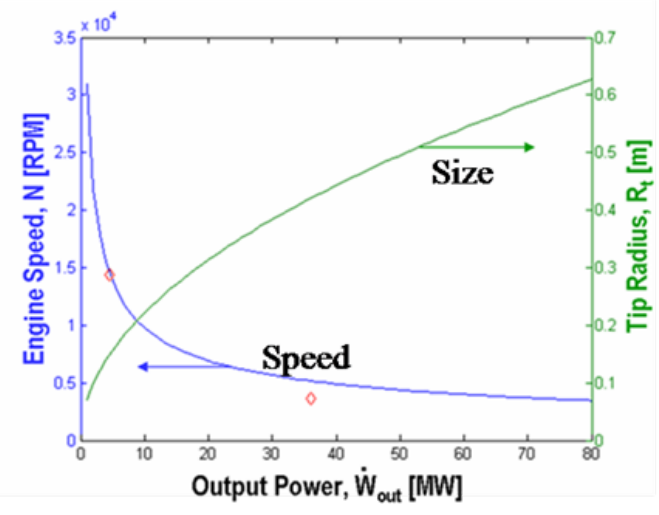

Figure 1. Gas turbine speed and size as a function of rated power.

Results of a design study to estimate size-reduction as a function of frequency, for several permanent-magnet generators, are summarized in Table I. These generators were optimized for size only. Fig. 2 shows these generators directly coupled to existing commercial gas turbines.

TABLE I.

\begin{tabular}{|c|c|c|c|c|}
\hline $\begin{array}{c}\text { Power } \\
(\mathrm{MW})\end{array}$ & $\begin{array}{c}\text { Speed } \\
(\mathrm{rpm})\end{array}$ & $\begin{array}{c}\text { Electric } \\
\text { Freq. } \\
(\mathrm{Hz})\end{array}$ & $\begin{array}{c}\text { Weight } \\
(\mathrm{kg})\end{array}$ & $\begin{array}{c}\text { Volume } \\
\left(\mathrm{m}^{3}\right)\end{array}$ \\
\hline 5 & 15,000 & 750 & 1700 & 2.34 \\
\hline 11 & 11,000 & 733 & 2700 & 7.25 \\
\hline 15 & 7,900 & 395 & 5000 & 13.23 \\
\hline 20 & 3,600 & 480 & 9000 & 33.37 \\
\hline 40 & 3,600 & 600 & 13030 & 38.4 \\
\hline
\end{tabular}

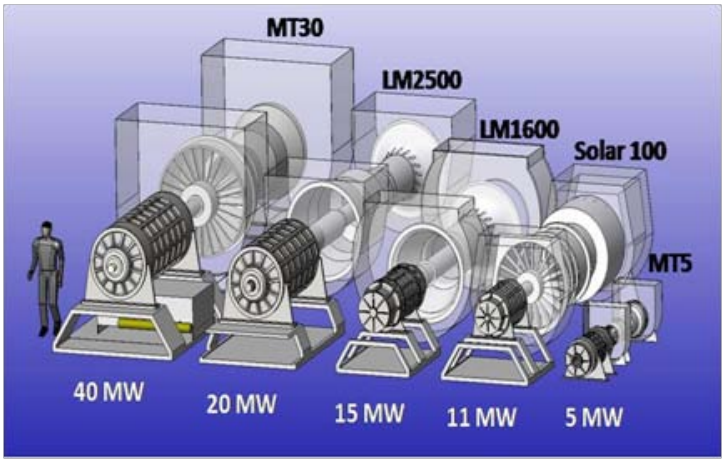

Figure 2. High-frequency PM generators directly coupled to existing gas turbines.

\section{High-frequency transformers}

The size of electric transformers is a function of frequency f. The transformer Wa product, a key transformer parameter that reflects size, varies as $1 /$ f. For multi-MVA transformers a significant size reduction can be achieved in power systems were several units are used. To quantify this gain at the power levels of interest, a detailed design study of high-frequency transformers is necessary. We plan to conduct such a study in the near future.

\section{HFAC POWER SYSTEM MODEL}

For a HFAC power system, a single frequency needs to be selected so that several generating units can be interconnected. While the frequency values for the gen-sets studied so far are favorable for reducing size, they are probably too high and not practical because of the high losses they would generate in the generation units and the distribution network. A frequency between $120 \mathrm{~Hz}$ and $400 \mathrm{~Hz}$ is more likely to provide an advantageous balance between size reduction and efficiency penalty.

A compromise frequency of $240 \mathrm{~Hz}$ has been selected based on preliminary analyses of size reduction and efficiency. A $240 \mathrm{~Hz}$ HFAC power system based on notional power system architecture has been developed in the Matlab\Simulink modeling environment. Top-level model setup is shown in Fig. 3 and component definitions are in Table II.

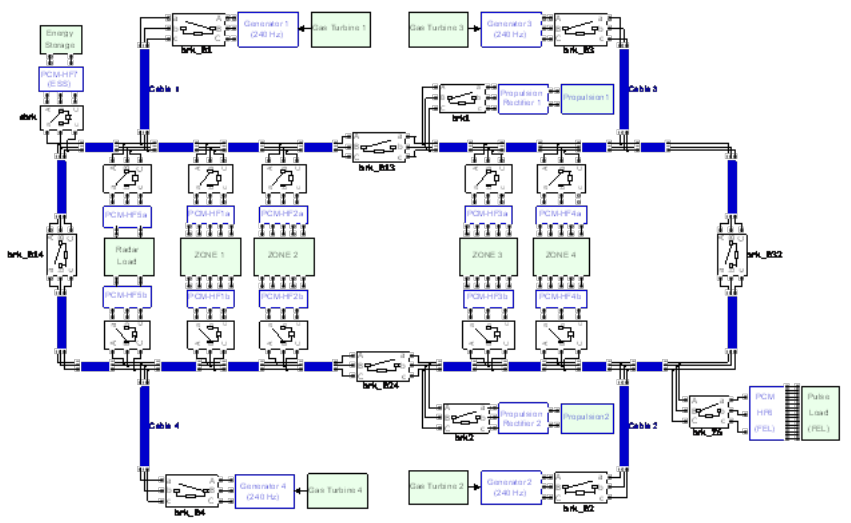

Figure 3. Matlab\Simulink HFAC power system model.

TABLE II.

\begin{tabular}{|c|c|}
\hline Generation power & $80 \mathrm{MW}$ \\
\hline Distribution frequency & $240 \mathrm{~Hz}$ \\
\hline Distribution voltage & $4.16 \mathrm{kV}$ \\
\hline Prime movers & $\begin{array}{l}\text { Gas turbines } \\
\cdot 2 \mathrm{x} 36 \mathrm{MW} / 3600 \mathrm{rpm} \text { twin-shaft } \\
\cdot 2 \mathrm{M} 4 \mathrm{MW} / \sim 14400 \mathrm{rpm} \text { single-shaft }\end{array}$ \\
\hline Generators & $\begin{array}{l}\text { Synchronous wound-field cylindrical rotor } \\
\cdot 2 \mathrm{x} 47 \mathrm{MVA} / 3600 \mathrm{rpm} / 8 \text {-pole } / 0.8 \mathrm{Pf} \\
\cdot 2 \mathrm{x} 5.25 \mathrm{MVA} / 14400 \mathrm{rpm} / 2 \text {-pole } / 0.8 \mathrm{Pf}\end{array}$ \\
\hline Turbo-generators & Direct-coupling (no gear boxes) \\
\hline Propulsion (x2) & $\begin{array}{l}\text { - 6-pulse passive rectifier } \\
\text { - Filter and voltage regulator/limiter } \\
\text { - Current-controlled hysteresis PWM inverter } \\
\text { - Permanent-magnet synchronous propulsionmotor } \\
\text { (36.5MW/120rpm) } \\
\text { - Propeller load }\end{array}$ \\
\hline Service loads & 4 Zones ( 22 total loads) \\
\hline Special load & Radar load \\
\hline Pulse load & Free Electron Laser (FEL) \\
\hline Energy storage & $100 \mathrm{MJ}$ (4 MW max) Super-Capacitor module \\
\hline Switch gears & $\begin{array}{l}\text {-3-phase AC breakers } \\
\text { - Semiconductor switches (DC breakers) }\end{array}$ \\
\hline
\end{tabular}




\section{DYNAMIC PERFORMANCE: PRELIMINARY ANALYSIS}

A fixed-time step of $10 \mu$ s was selected as a compromise between an adequate resolution of transients and a reasonable model execution time on a desktop PC. The model is numerically stable as various loads are switched on and off.

Initial model verification results showed that the predicted steady state values of voltages, currents, and powers at various load terminals are as expected. Fig. 4 shows the ring bus voltage with the expected $240 \mathrm{~Hz}$ frequency and $5.9 \mathrm{kV}$ peak voltage for a $4.16 \mathrm{kV}$ (line-line) bus.

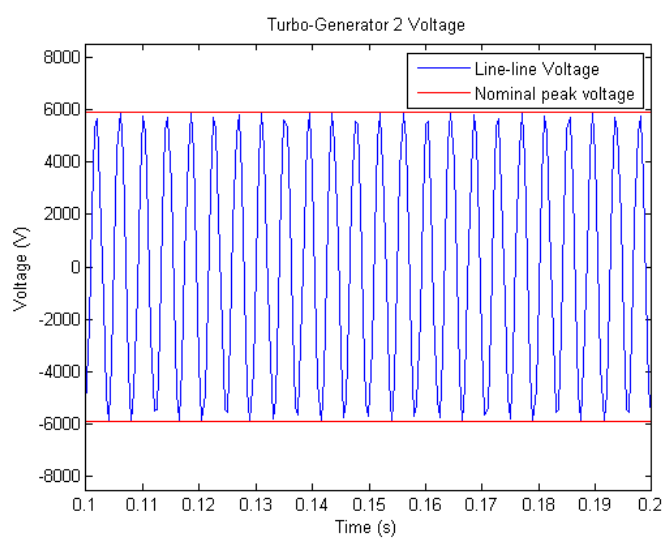

Figure 4. Model verification: Ring bus phase-voltage and frequency.

The goal of the initial phase of this analysis effort was to assess the ability of the model to predict the dynamic performance of the HFAC power system. To that end, four case studies were conducted. Brief descriptions and initial results are discussed in the next sections.

\section{A. Case study 1: Partial loss of generation}

In the first simulation, one of the large generators is dropped out of the power system during full operation by suddenly opening its breaker as depicted in Fig. 5.

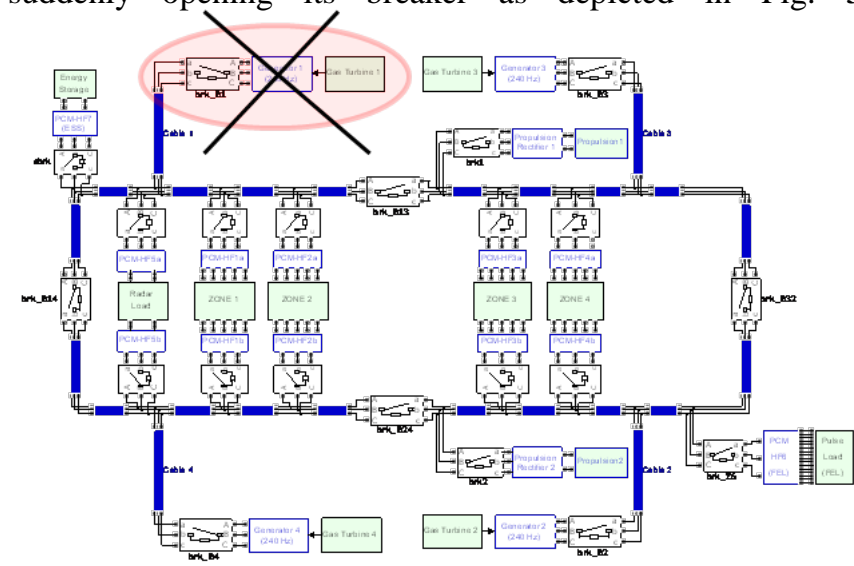

Figure 5. Model for case study with dropped generator.

Fig. 6 shows the four gas turbines' dynamic responses. As expected, the speed of the dropped turbo-gen increases just after its breaker is opened. The remaining three turbines stay under control while assuming the dropped load, causing their share of the load to increase (Fig. 7) and their speeds to drop.

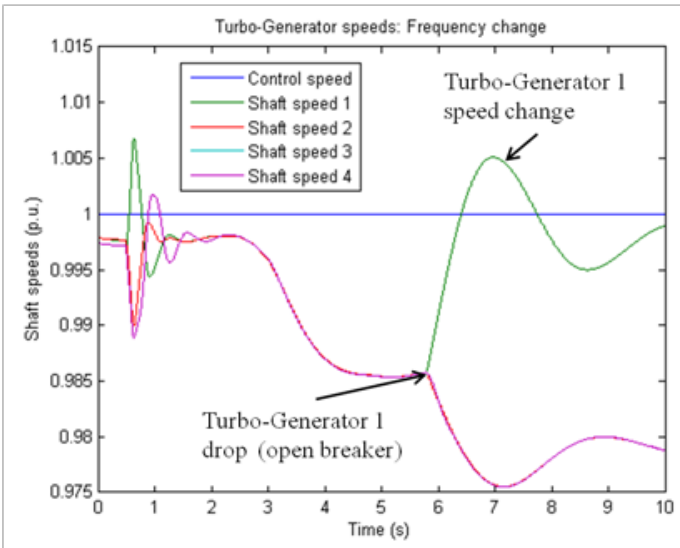

Figure 6. Gas turbines' dynamic response after loss of a generator.

Fig. 7 shows that load sharing among the remaining turbogenerators is quickly re-established after the event and that the system remains under control afterwards. In addition, bus voltage drops during the event but recovers quickly as depicted in Fig. 8.

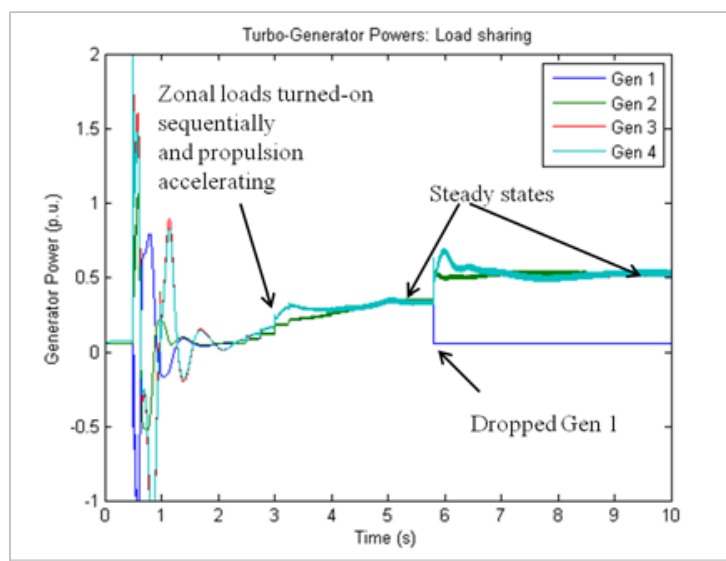

Figure 7. Load sharing dynamics after loss of a generator.

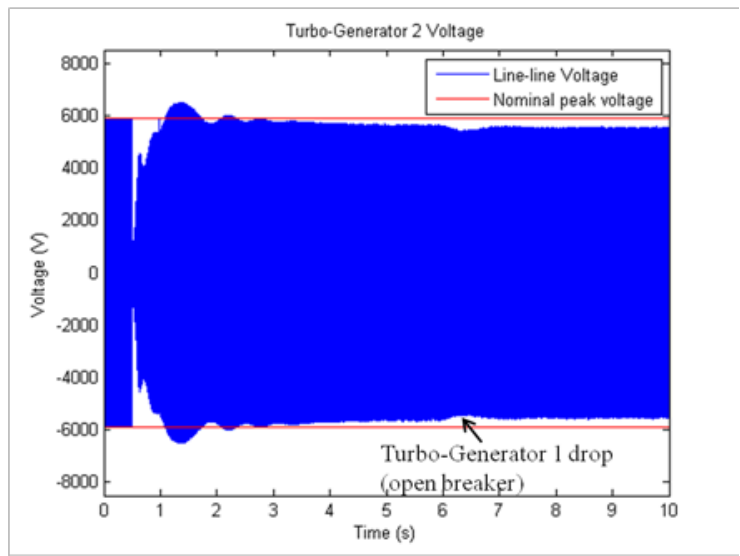

Figure 8. Voltage change after loss of a generator. 


\section{B. Case study 2: Application of a large step load}

In the second case study a large step load provided by a free electron laser system (FEL) is applied. Prior to applying the step load, the power system is operating normally, supplying various service loads and propulsion loads with a total output power of $\sim 25$ MW ( $\sim 25 \%$ MVA capacity). The FEL is rather a complicated system and was included in the HFAC architecture to highlight the challenges of modern systems planned for future all-electric ships. It includes several sub-systems that require various power levels and power types with several power conditioning modules. Some sub-components require permanent power from kilowatts to few megawatts while the pulse requires $\sim 25$ MW for few seconds. In the experiment, turbines' response, bus voltage, and generator load sharing are monitored when the FEL load is activated. The results are presented in the following graphs.

Fig. 9 shows the gas turbines' dynamic response. The speeds of the four turbo-generators drop but remain in synch as the controller was able to maintain them under control. A droop-control was applied to the four turbines with a $4 \%$ droop each. The observed frequency drop is $\sim 2 \%$.

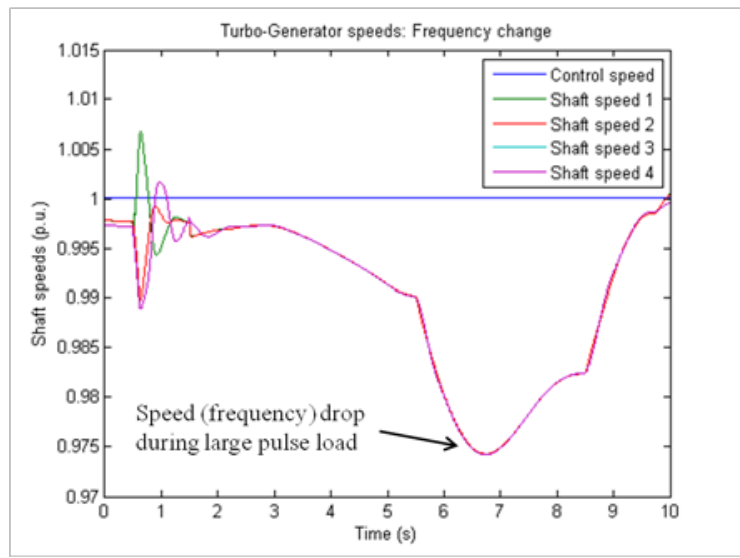

Figure 9. Gas turbines' dynamic response when large step load is applied.

Initial load sharing among the four turbo-generators is reestablished relatively quickly after the large step load is applied, as shown in Fig. 10. The light-green curves in Fig. 10 are those of the smaller turbo-generators.

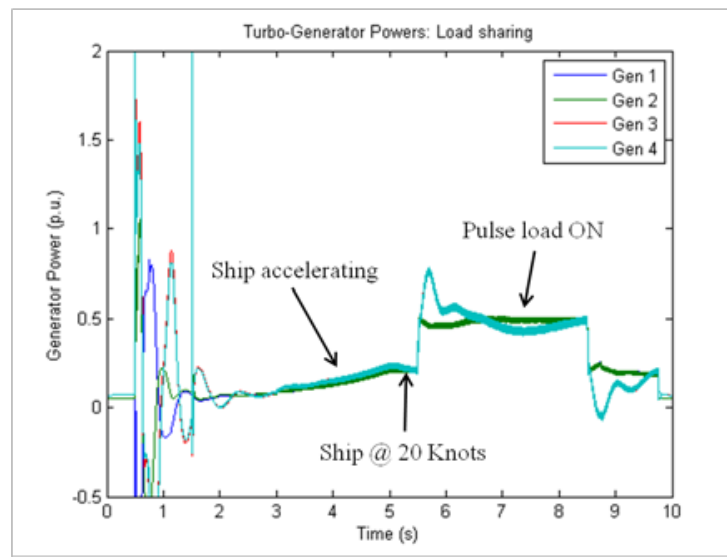

Figure 10. Turbo-generator load sharing when large step load is applied.
As can be seen in Fig. 11, the voltage also drops during the large step load. Voltage amplitude drops by $\sim 8 \%$, indicating that voltage control parameters may need to be re-adjusted to maintain voltage close to rated value so that other loads are not affected. Perhaps, it is worthwhile to note that using this modeling and simulation approach, and case studies similar to the ones just described, the designers of the control system can fine-tune the control parameters by running several numerical experiments until they achieve the desired outcome, with confidence, before a prototype control system is built and tested.

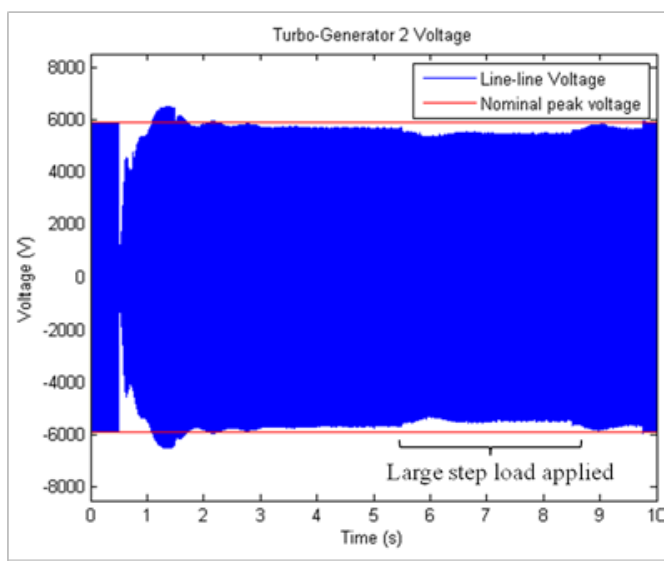

Figure 11. Voltage change when large step load is applied.

\section{Case study 3: Restoration of power to a vital load}

In the third case study a scenario simulating a fault causing a vital load to lose power momentarily is considered. The challenge is to determine whether power can be transferred quickly, and in a controlled manner, to the vital load. This represents a power system re-configuration scenario and allows performance assessment of the architecture under study with respect to this important power system property.

The case starts by the loss of a generator as in the first case with the bus segment connected to the faulty generator isolated from the rest of the system as shown in red in Fig. 12.

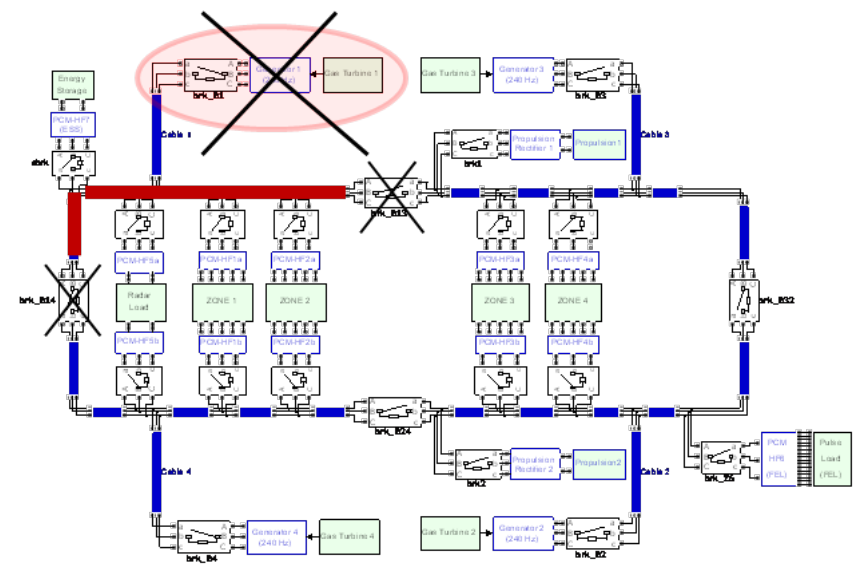

Figure 12. Condition with disabled ring bus segment (red).

In this architecture all zonal and radar loads are fed from two different segments of the distribution ring-bus. The vital 
load considered here is the radar load which requires few megawatts. As stated earlier, the goal of the exercise is to determine whether the power system remains under control, and with no detrimental effects to other system components, when various switches and breakers are turned on and off to, first, cut power to the load and then restore it using a different supply bus segment and different power conditioning modules. The timing of various switches and breakers operations is at the discretion of the designer or analyst running the simulation. A study requiring several simulation runs can be conducted, for example, to determine various time delay intervals for which the power system remains under control or becomes unstable. In this exercise the ring bus breakers isolating the faulty segment were opened $3 \mathrm{~ms}$ after the generator fault is initiated, 2 ms later the upper radar load breaker is opened to further isolate it, and after another $3 \mathrm{~ms}$, the lower breaker connected to a working bus segment is closed to restore power to the radar load. Fig. 13 shows the radar load dc voltage as power is switched from one bus to another. Time intervals used here are for demonstration only and correct and realistic values require knowledge of the properties of breakers and switches used in the experiments.

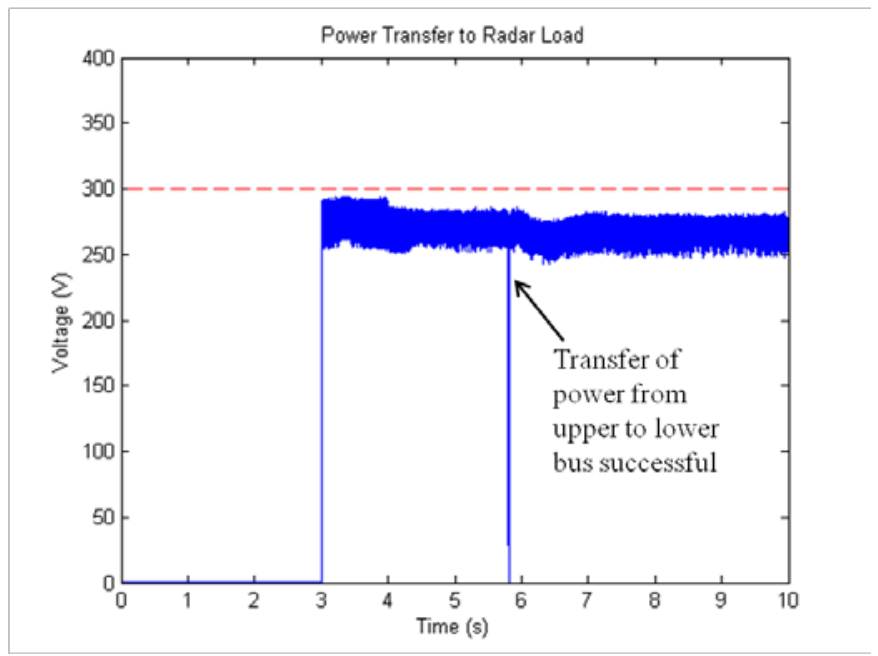

Figure 13. Radar load dc voltage before and after power transfer.

Under the conditions of this demonstration exercise no major disruption in other parts of the system were observed during the power transfer event. More detailed reconfiguration studies will be conducted in the near future using this power system model.

\section{Case study 4: Operation of energyc storage system}

A $100 \mathrm{MJ}, 4 \mathrm{MW}$, super-capacitor energy storage module is used with the HFAC power system to perform UPS or load leveling functions. The energy storage system (ESS) is directly connected to the ring bus as shown highlighted in Fig. 14. The initial aim of this case study is to verify proper operation of the energy storage system within the HFAC power system model. The ESS is used as a UPS unit supplying power to a load after a loss of power. The case setup is similar to the previous one except that power from the
ESS is supplied to the load through an isolated segment of the ring-bus.

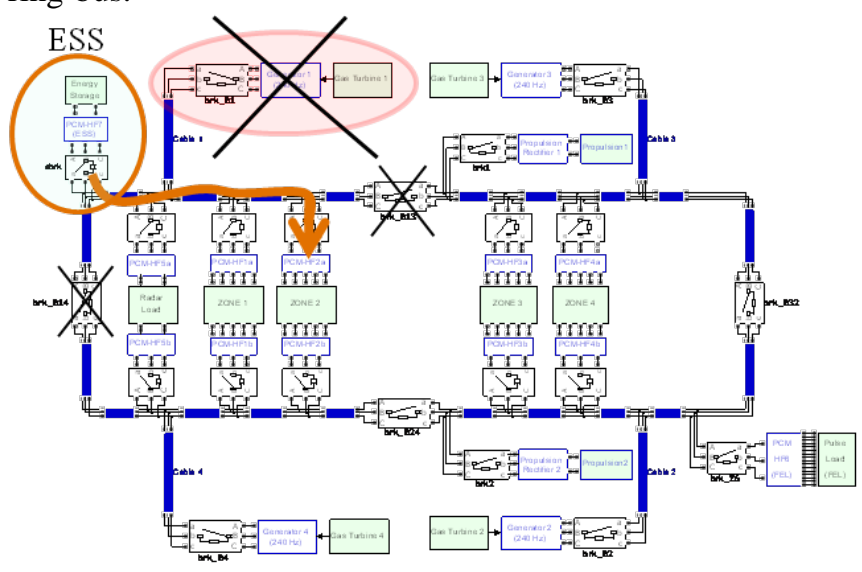

Figure 14. Set-up for case study 4 with ESS module highlighted in orange.

In this scenario, the loss of a generator causes the isolation of a bus segment resulting in a loss of power to several connected loads. The ESS is activated after a certain time delay to, in effect, replace the lost generator but at a lower power level. Fig. 15 shows the output power of the ESS along with the total connected power of $\sim 3.2 \mathrm{MW}$ which agree well. The ESS is set-up to supply the loads connected to its terminals at any power level up to $4 \mathrm{MW}$.

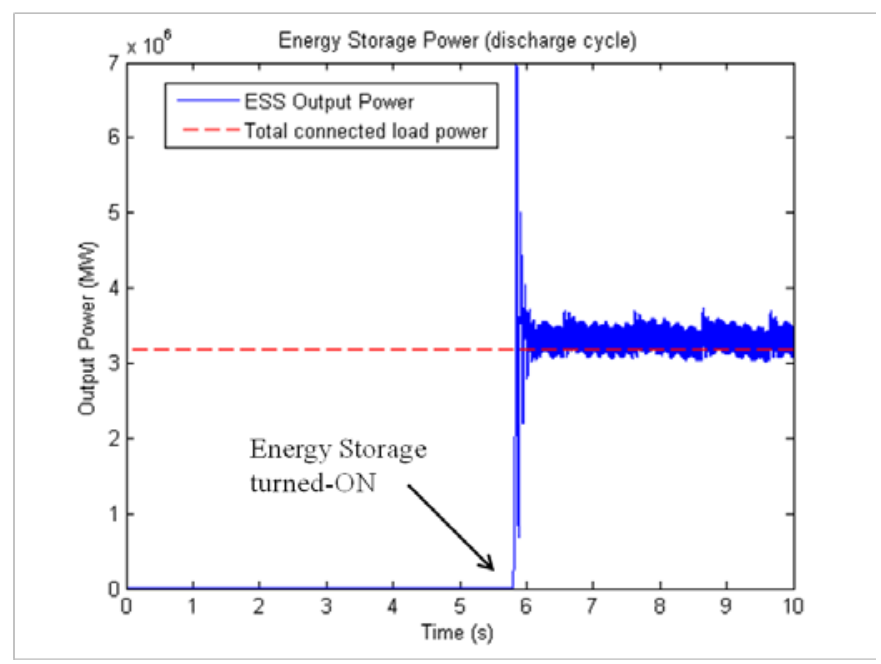

Figure 15. ESS output power and connected load power.

The 4-second duration of the ESS operation causes the voltage to drop to the expected level. This is shown in Fig. 16. It is important to note that in this example, the ESS was connected to an isolated segment of the main ring-bus and, consequently, there was no need to synchronize its output with the other generators before the connection. In future case studies we will consider situations where the ESS is connected to the ring bus in parallel with operating generator-sets, thereby requiring synchronization. 


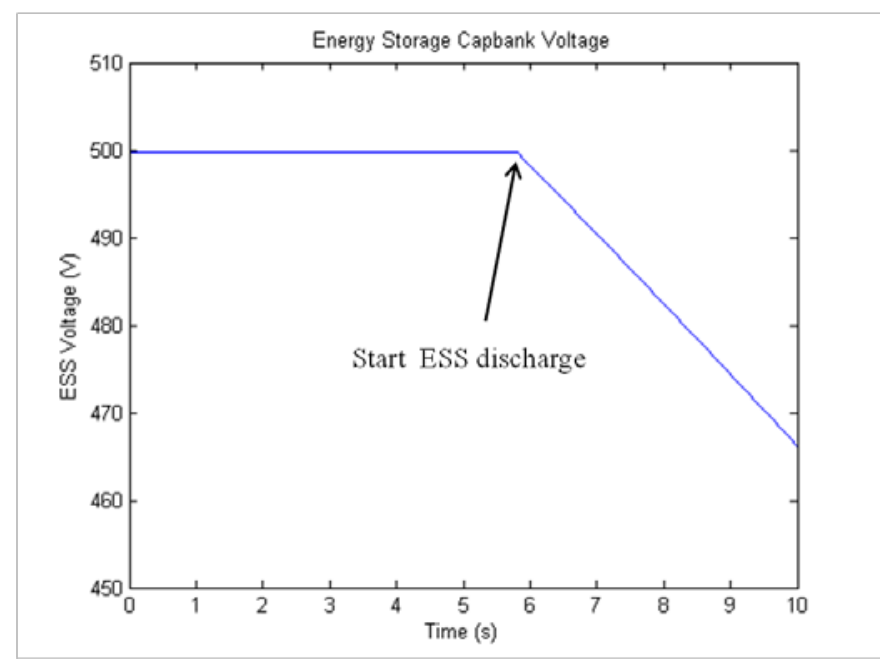

Figure 16. ESS voltage during dischage.

\section{SUMMARY}

This work shows that a $240 \mathrm{~Hz}$ system may be a promising approach to reduce the size of the power system in future ships. To assess that the electric performance can be acceptable, a dynamic model of a high-frequency power system (HFAC) for an all-electric ship has been developed in the Matlab\Simulink environment. Realistic performance scenarios were simulated in four different case studies. The model is numerically stable and initial results of the test cases are encouraging. Detailed analyses are planned in the near future to further assess its performance with respect to other power system architectures.

\section{REFERENCES}

[1] K. Pesyna, R. Hebner, A. Ouroua, R. Thompson, and E. Bowles, "High-speed generator options for direct coupled gas turbine flight and ground based power system", presenterd at the $8^{\text {th }}$ Annual Directed Energy Professional Society Symposium, Hawaii, USA 2005.

[2] S. Vijlee, "Optimizing a system of gas turbine engines and generators for marine power generation," Master thesis, The University of Texas at Austin, April 2005.

[3] S. Vijlee, A. Ouroua, L. Domaschk, and J. Beno, "Directly-coupled gas turbine-permanent magnet generator sets for prime power generation on board electgric ships”, IEEE, Electric Ship Technology Symposium, May 21-23, 2007, Arlington, VA, USA.

[4] R.M. Calfo, M.B. Smith, and J.E. Tessaro, "High-speed generators for power dense, medium power, gas turbine generator sets”, Naval Engineer Journal, no.2, 2007, pp. 63-81. 